\title{
Retesting the influences of mutation accumulation and antagonistic pleiotropy on human senescence and disease
}

\author{
Erping Long ${ }^{1,2}$, Jianzhi Zhang ${ }^{1, *}$ \\ ${ }^{1}$ Department of Ecology and Evolutionary Biology, University of Michigan, Ann Arbor, Michigan, \\ USA \\ 2State Key Laboratory of Ophthalmology, Zhongshan Ophthalmic Center, Sun Yat-sen University, \\ Guangzhou, China.
}

\section{To the Editor}

Multiple hypotheses have been proposed to explain the origin of senescence, which refers to the gradual deterioration of functional characteristics with ageing ${ }^{1}$. Two of the leading hypotheses are mutation accumulation (MA) ${ }^{2}$ and antagonistic pleiotropy (AP) ${ }^{3}$. The MA hypothesis states that natural selection is ineffective in purging mutations causing senescence because these mutations have little fitness consequence due to the absence of phenotypic effects until after the reproductive age. The related AP hypothesis asserts that these mutations may even be selectively favored because they promote growth or reproduction owing to their pleiotropic effects. By examining genetic variants associated with diseases appearing at different life stages, Rodriguez and colleagues claimed to have found genomic evidence for these two hypotheses in humans ${ }^{4}$. Our further analyses of their data, however, show that their results are unrobust and conclusions unsupported.

In testing the MA hypothesis, Rodriguez et al. took advantage of published genome-wide association studies and analyzed 104 single nucleotide polymorphisms (SNPs) associated with 46 diseases. They reported that risk allele frequencies (RAFs) are significantly higher for the SNPs associated with late-onset diseases than those associated with early-onset diseases when the threshold between the early- and late-onset is placed anywhere at or before the age of 40 yrs (Fig. 1a in Rodriguez et al. ${ }^{4}$ ), a trend predicted by the MA hypothesis under the assumption of mutation-selection balance ${ }^{5}$. However, this trend disappears (Fig. 1a) upon the removal of only one disease-acute lymphoblastic leukemia (ALL), which has an age onset of $1 \mathrm{yr}$ and is associated with 9 SNPs with exceptionally low RAFs (all < 0.06, compared with the mean RAF of 0.31 in the entire data). Rodriguez et al. further showed that the mean genetic variance explained by disease-associated SNPs is significantly greater for the late-onset diseases than early-onset diseases when the age threshold is placed at or before 36 yrs (Fig. $1 \mathrm{~b}$ in Rodriguez et al. ${ }^{4}$ ). But, when ALL is excluded, the above difference vanishes almost entirely; only when the age threshold is at

*Correspondence to: Jianzhi Zhang, Department of Ecology and Evolutionary Biology, University of Michigan, 4018 Biological Science Building, 1105 North University Avenue, Ann Arbor, MI 48109, Phone: 734-763-0527, Fax: 734-763-0544, jianzhi@umich.edu. 
20,22 , or 24 yrs is there a significant difference (Fig. 1b). Because this observation is hard to interpret and because the statistical significance does not hold upon the correction for multiple testing, the age-related difference is unlikely to be genuine. Rodriguez et al. would have found these problems if they had investigated the robustness of their results by bootstrapping the diseases ${ }^{6}$. At any rate, their findings appear idiosyncratic and their evidence for the MA hypothesis is unrobust.

In testing the AP hypothesis, Rodriguez et al. compiled SNPs that are each associated with two diseases. They then divided these SNPs into four groups on the basis of whether the same allele of a SNP (or two strongly linked alleles at two SNPs) increases the risks of both diseases and whether the two diseases occur in the same life stage. Using a $2 \times 2$ table, they found a significant excess of SNPs (26 in total) for which the two alternative alleles respectively increase the risk of one early-onset and one late-onset disease (Fig. 1c in Rodriguez et al. ${ }^{4}$ ), which they interpreted as evidence for the AP hypothesis. However, the key prediction of the AP hypothesis is that the risk allele for the early onset-disease, which is also the protective allele for the late-onset disease, is selected against relative to the alternative allele at the SNP. That is, under the mutation-selection balance, the AP hypothesis predicts a lower RAF for the early-onset disease than for the late-onset disease for each of these 26 SNPs. To verify this prediction, we examined the CEU population genomic data from Phase 1 of the 1000 Genomes Project ${ }^{7}$. As shown in Fig. 1c, only 35\% of the 26 SNPs are consistent with the AP hypothesis. To examine the robustness of this finding, we limited the analysis to the subset of 26 SNPs for which the RAFs for the two diseases differ by at least $\delta=0.05,0.10,0.15,0.20$, or 0.25 , respectively. We found that, in all cases, only $28 \%$ to $35 \%$ of the SNPs are consistent with the AP hypothesis (Fig. 1c). While none of these percentages are significantly below the chance expectation of 50\% ( $P>$ 0.05 , binomial test followed by multiple testing correction) to allow the rejection of the AP hypothesis, there is also no evidence for the AP hypothesis.

We note that the above tests of the MA and AP hypotheses rely on the assumption that the mutational supply of deleterious alleles at a SNP is counterbalanced by the selective purge of such alleles. Neither hypothesis could be tested in this fashion when the mutationselection balance is violated, which is possible, for example, due to recent demographic or environmental changes ${ }^{8}$. In summary, our reanalysis of Rodriguez et al.'s data found no evidence for the MA or AP hypothesis. More studies are needed to further test the influences of MA and AP in human senescence and age-related disease. In particular, because the fitness effect varies tremendously among diseases and disease subtypes and because the mutation-selection balance may not hold for all disease alleles, it is necessary to consider these factors in future studies. With the rapid progress of medical genomics, data size may soon become sufficiently large to permit robust tests of various evolutionary hypotheses of senescence, a subject that is increasingly important due to the disproportional expansions of our ageing populations over time ${ }^{9}$.

\section{References}

1. Trindade LS et al. Front Genet 4, 25, (2013). [PubMed: 23508239]

2. Medawar PB An Unsolved Problem of Biology. (H.K. Lewis \& Co., 1952). 
3. Williams GC Evolution 11, 398-411, (1957).

4. Rodriguez JA et al. Nat Ecol Evol 1, 55, (2017). [PubMed: 28812720]

5. Hartl DL \& Clark AG Principles of Population Genetics, 4th Edition. (Sinauer Associates, Inc., 2007)

6. Efron B \& Tibshirani R An Introduction to the Bootstrap. (Chapman \& Hall/CRC, 1993).

7. Abecasis GR et al. Nature 491, 56-65, (2012). [PubMed: 23128226]

8. Wachter KW, Steinsaltz D, Evans SN. Proc Natl Acad Sci U S A. 111, 10846-10853, (2014) [PubMed: 25024186]

9. United Nations Department of Economic and Social Affairs, World Population Ageing (2015). 

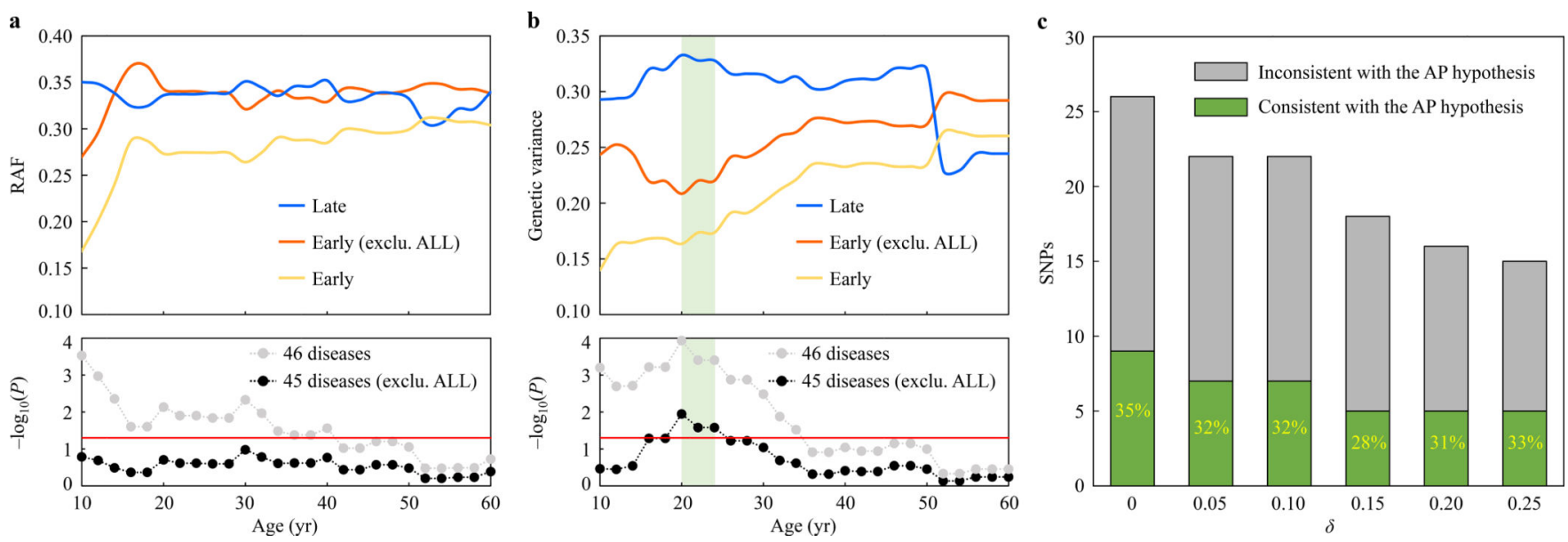

Figure 1. Retesting the influences of MA and AP on human senescence and disease.

a, Mean risk allele frequencies (RAFs) for SNPs associated with early- (yellow) or lateonset (blue) diseases, as a function of the age threshold between these two groups of diseases. The result for early-onset diseases upon the removal of acute lymphoblastic leukemia (ALL) is shown in orange. Grey dots depict the $-\log _{10}(P$-value $)$ at each age threshold for the whole data (Wilcoxon one-tail test), whereas black dots depict the corresponding values upon the removal of ALL. The red horizontal line shows $-\log _{10}(0.05)$. b, The same as a, but showing the mean genetic variance explained by the SNPs in each group of diseases. Upon the removal of ALL, significant differences are observed only for thresholds at 20,22, or 24 yrs (green shading), and they disappear upon multiple testing correction. c, Number (and percentage) of SNPs consistent (green) or inconsistent (grey) with the AP theory, as a function of the minimal difference $(\delta)$ between the frequencies of the two alternative alleles at each SNP. All analyses followed Rodriguez et al. ${ }^{4}$ unless otherwise mentioned. Panels $\mathbf{a}$ and $\mathbf{b}$ are drawn in the same fashion as Figure 1ab in Rodriguez et al. ${ }^{4}$ for easy comparison (adapted with permission). 